\title{
NEW INSIGHTS ON UNIFICATION OF RADIO-LOUD AGN
}

\author{
MARCO CHIABERGE \\ Istituto di Radioastronomia - CNR \\ via P. Gobetti 101 \\ 40129 Bologna, Italy \\ E-mail: chiab@ira.cnr.it
}

\begin{abstract}
The radio-loud AGN unification model associates powerful radio galaxies with radio-loud quasars and blazars. In analogy with the radio-quiet scheme, the nuclear regions of objects showing only narrow emission lines in their optical spectrum are thought to be obscured to our line-of-sight by a geometrically and optically thick dusty "torus". In objects showing broad emission lines we directly observe the innermost parsecs around the central black hole, i.e. the broad line region and the accretion disk. Radiation from the base of the relativistic jet dominates the overall emission of blazars, that are seen almost pole-on. Although the broad picture seems to be well established, there are several fundamental aspects that are still to be understood. HST studies have recently shed new light on many issues, from the properties of the nuclei to the structure of the host galaxies.
\end{abstract}

\section{The standard unification picture}

The standard picture for unification of both radio-quiet and radio-loud AGN is based on the anisotropy of the nuclear emission: objects intrinsically identical appear different to observers located along different viewing directions. In addition to the presence of 1-to-100pc-scale absorbing tori, radio-loud AGN have two powerful relativistic jets, which constitute a further source of anisotropy. The jets emerge from the innermost regions, and propagate along the rotation axis of the accretion disk formed around the central black hole ${ }^{38,4}$. The torus blocks the direct view of both the accretion disk and the surrounding broad line region (BLR) to observers located perpendicularly to the jet axis. The extended (kpc-scale) narrow emission line region (NLR) is visible to such an observer, which would classify that object as a narrow-line radio galaxy (NLRG). If the line-of-sight forms a very small angle to the jet axis, the emission is dominated by non-thermal 
radiation from the jet, which is strongly boosted by relativistic effects ${ }^{\mathrm{a}}$. In that case, such object would appear to us as a blazar, i.e. either a flat spectrum radio quasar (FSRQ) or a BL Lac, depending on whether strong emission lines (EW > $5 \AA$ ) are present or absent, respectively. For intermediate viewing angles $(\theta>1 / \Gamma)$, where the jet radiation appears less boosted or even de-boosted, both the accretion disk and the BLR become visible, and the object appears to us as a steep spectrum radio quasar (SSRQ) or, for lower nuclear luminosities, a broad line radio galaxy (BLRG).

Among the various observations that can be performed to prove the validity of such a unification scenario, the detection of a hidden BLR seen through scattered radiation is considered as one of the most solid and direct tests $^{2,1}$. In fact, scattering mirrors provide a "periscope" for viewing the nucleus from other directions

\subsection{Open problems}

The "zeroth-order" unification scheme described above generally accounts for the observational properties of radio-loud AGN. However, several crucial issues are still open, and need further investigation. First of all, there is clear evidence that such a scheme must be split into two different models, in order to account for unification of low and high power objects separately. At high luminosity, radio galaxies with "edge-brightened" (FR II) morphology are unified with quasars (SSRQ and FSRQ), while for lower powers, radio galaxies with "edge-darkened" morphology (FR I) are believed to be the parent population of BL Lac objects. The need for two different schemes comes from the "absence" of strong emission lines in both BL Lacs and FR I, while strong, high excitation lines are a common characteristic of powerful FR II and quasars. Furthermore, in the low-power scheme, the "intermediate" class analogous to BLRG and SSRQ appears to be missing, since only very few examples of broad-lined FR Is are known (see section 5). Thus, the nuclear structure of low and high power objects may differ in some crucial aspect.

Let me now summarize what are, in my opinion, the most important open problems. i) The lack of significant broad emission lines in FR I and BL Lacs implies that, in the framework of the unification model, there is no need for the presence of obscuring tori in these sources. However, molecular

\footnotetext{
a The observed integrated flux is enhanced by a factor $\delta^{4}, \delta=[\Gamma(1-\beta \cos \theta)]^{-1}$ is the beaming factor, $\theta$ is viewing angle to the jet axis, and $\Gamma$ and $\beta$ are the bulk Lorentz factor and velocity of the jet, respectively.
} 
tori are thought to be present in all other AGN, and they are also believed to function as a fuel reservoir for the active nucleus; ii) environment: FR Is and BL Lacs seem to prefer different environments. FR I are predominantly found in high density clusters ${ }^{45}$, while BL Lacs seem to avoid rich clusters ${ }^{30}$; iii) a few BL Lacs show faint (and variable) broad lines ${ }^{15}$; iv) a fraction of the BL Lac population shows a radio morphology more typical of $\mathrm{FR}^{2} \mathrm{II}^{26}$; v) a substantial fraction of narrow-lined FR II have atypical low-ionization optical spectra $\left(L_{[O I I I]}<L_{[O I I]}\right)$ similar to those of FR I, while quasars have $L_{[O I I I]}>L_{[O I I]}$; vi) although polarized broad emission lines have been observed in a few NLRG (e.g. 3C 234), it is still unclear what is the incidence of such a phenomenon among radio-loud AGN. Furthermore, in many objects the origin for polarization is not clear: scattering and dichroic extinction are two possible interpretations ${ }^{14}$; vii) the quasar fraction in complete samples of radio galaxies is strongly dependent on luminosity ${ }^{43}$, but it is unclear whether this happens because the inner radius of the torus increases for increasing luminosity of the central quasar, or because of the rise of a distinct population of "starved" quasars at low luminosities.

These "phenomenological" issues translate into more general questions on the physical properties of radio-loud AGN: are FR I and FR II (or BL Lacs and quasars) physically different? Are their central BH masses different? What are the properties of accretion around the BH? Are their jets different? Do all RL AGN have a BLR? In the following I will try and summarize the current observational scenario.

\section{Jets in radio galaxies and quasars}

Relativistic jets are seen emerging from the very innermost regions of the active nucleus, on sub-pc scales ${ }^{25,20}$. Radio observations show that jets are relativistic in both FR I and FR II radiogalaxies on parsec scales. Superluminal motions of radio components are observed in a large number of quasars and, although less prominently, also in BL Lacs. In low power radio galaxies, while proper motions of radio components are commonly observed on small scales, the jet slows down to sub-relativistic velocities over distances of $\sim 1-10 \mathrm{kpc}^{27}$. Superluminal motions of optical components have also been observed with the HST in M 87, having apparent speed in the range $4-6 c$ thus strongly supporting unification with BL Lacs ${ }^{5}$.

Recently, significant step forward in our knowledge of the structure of jets has been taken. In particular, high resolution radio data have shown the presence of transverse structures in jets associated with both low and 
high power radio galaxies, and even on the VLBI scale" ${ }^{21}$ Their "limbbrightened" appearance is currently explained as due to the presence of velocity structures in the jet: a fast, highly relativistic "spine" $(\Gamma \sim 15)$ and a slower external layer which moves at a slower speed, possibly because of the interaction with the surrounding ambient medium. In FR II such structures appear to persist on very large scales (>> 10kpc, e.g. 3C $353^{34}$ ), indicating that at least the "spine" of FR II jets does not suffer substantial deceleration. Independent evidence for large-scale high-speed jets in quasars and FR II is provided by X-ray Chandra observations. X-ray emission from large-scale extragalactic jets is best interpreted as a result of inverse Compton emission from relativistic particles scattering off seed photons of the cosmic microwave background ${ }^{36,8}$. Such model requires the bulk Lorentz factor of the jet (spine) to be $\sim 15$.

\section{Host galaxies}

A fundamental prescription of the unification models is that unified classes must share the same properties as far as the extended (unbeamed) characteristics are concerned. Therefore, the properties of the host galaxy are crucial parameters for testing unification scenarios. Quasar hosts spanning a range of redshift $z=0.1-2$ have been extensively studied with the $\mathrm{HST}^{16}$. Radio loud QSO are hosted by bright $\left(L>L^{*}\right)$ massive ellipticals, which are similar in magnitude and morphology to radio galaxies hosts. The same result holds for BL Lacs host galaxies, which appears to be similar to FR I hosts, in substantial agreement with the unification scenario ${ }^{39,33}$. However, the absence of BL Lacs in rich cluster environments ${ }^{30}$ and the lack of dust lanes in BL Lac hosts, compared with those of FR Is in which dust lanes are ubiquitous, are still issues to be addressed.

\section{Black hole masses}

The correlations between the mass of the central black hole and some fundamental parameters of the host galaxy (either the central stellar velocity dispersion $\sigma$ or the optical magnitude of the bulge $\left.L_{\text {bulge }}\right)^{37,29}$ are considered as powerful tools to estimate $\mathrm{M}_{\mathrm{BH}}$ within an accuracy of a factor 2-3. But the determination of the velocity dispersion and/or the optical magnitude of the host is not always straightforward, especially if a strong nuclear component is present, such as in the case of quasars and BL Lacs. BH masses of BL Lacs have been estimated by ${ }^{3,17}$ using the $\mathrm{M}_{\mathrm{BH}}-\sigma$ relation, obtaining values in the range $5 \times 10^{7}-1 \times 10^{9} \mathrm{M}_{\odot}$. The distributions of 
central BH masses in BL Lacs and radio galaxies (of all species) appear to be consistent, thus supporting unification. However, to the best of my knowledge, a detailed comparison of well defined and statistically complete samples has not been performed yet.

Concerning high power objects, the BH mass of a sample of radio loud QSO and radio galaxies has been estimated using HST images of their host galaxies and the $\mathrm{M}_{\mathrm{BH}}-L_{\text {bulge }}$ relation ${ }^{29}$. These objects share a common range in $M_{\mathrm{BH}}$ but, interestingly, it appears that radio loud AGN have $\mathrm{BH}$ masses confined to $\mathrm{M}_{\mathrm{BH}}>10^{9} \mathrm{M}_{\odot}$. However, other authors do not find any relationship between radio-loudness and central black hole mass ${ }^{44}$.

\section{The HST view of FR I and FR II nuclei: implications for unification}
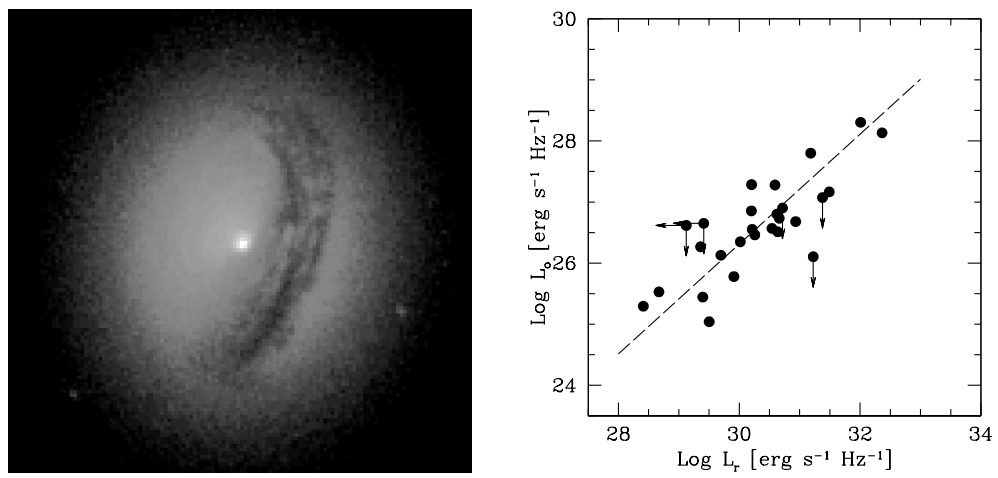

Figure 1. Left: the central 6 arcsec of 3C 449 as seen with HST/WFPC2. Right: optical CCC luminosity versus radio core luminosity for 3CR FR Is.

FR I: Optical nuclear studies of radio galaxies provide us with crucial information on the physical processes at work in their central regions. Furthermore, a direct test of the unification scheme, based on the nuclear emission, can be performed. HST has allowed us to study faint unresolved sources that are present in the center of most FR Is from the $3 \mathrm{CR}$ catalog ${ }^{9}$ (but see also other work $\left.{ }^{7,41}\right)$. We found that these Central Compact Cores (CCC) show a tight correlation with the radio core emission (both in flux and luminosity) which strongly argues for a common synchrotron origin from both components (Fig. 1). The detection of CCCs in $85 \%$ of the complete sample indicates that we have a direct view of the innermost nuclear regions of in 
the vast majority of FR I. Thus it appears that a "standard" geometrically and optically thick torus is not present in low luminosity radio galaxies. Any absorbing material must be distributed in a geometrically thin structure (thickness over radius ratio $\lesssim 0.15$ ) or, alternatively, thick tori are present only in a minority of FR I. The CCC fluxes are upper limits to any thermal disk emission. This implies extremely low radiative efficiency for the accretion process. The observed CCC emission corresponds to $\lesssim 10^{-7}$ $10^{-4}$ of the Eddington luminosity for a $10^{8} M_{\odot}$ black hole, which appears to be typical for these radio galaxies. This might also explain the lack of strong photo-ionized emission lines in the optical spectra of both FR Is and BL Lacs. The picture which emerges is that the innermost structure of FR I radio galaxies (and thus also of BL Lacs) differs in many crucial aspects from that of the other classes of AGN; they lack the substantial BLR, tori and thermal disk emission, which are instead associated with all other active nuclei.

A fine test for such a scenario has been obtained in the case of $\mathrm{M} \mathrm{87,a}$ famous and relatively powerful FR I radio galaxy ${ }^{32,42}$. M 87 was observed in the infrared at $10 \mu \mathrm{m}$ with the Keck and Gemini telescope, and no significant mid-IR nuclear excess is found. This supports the absence of a hidden quasar-like accretion process in the nucleus, which would heat the surrounding obscuring dust, thus producing a strong thermal IR excess, when compared to the optical observed flux. Of course it would be very interesting to extend this analysis to complete samples.

In two cases (Centaurus A and NGC 6251) the nuclear spectral energy distribution (SED) has been derived from the radio to the gamma-ray band. The SEDs show two broad peaks, very similar to those observed in BL Lacs and usually interpreted as non-thermal synchrotron and inverse Compton emission $^{22}$. The SED has been modeled in the framework of synchrotron self-Compton emission ${ }^{13,12,23}$, obtaining physical parameters for the source that are completely in agreement with those of BL Lacs of similar total power, thus quantitatively supporting the unification scenario. Furthermore, we found evidence that the emitting region in FR I has a slower bulk Lorentz factor $(\Gamma \sim 2)$, when compared to BL Lacs. This can be interpreted as a signature of the presence of velocity structures in the jet, e.g. a fast spine and a slower (but still relativistic!) layer similar to those observed in radio VLBI images. The spine dominates the emission in BL Lacs, while the slower layer is visible only when the jet direction forms a large angle to the observer's line-of-sight.

But are all FR I "starved quasars"? In fact, very few "broad-lined" 
FR Is, showing the signature of radiative efficient accretion and substantial BLR, are known. A famous example is 3C 120, which is associated to a peculiar S0 galaxy ${ }^{35}$. A few other FR I quasars have been recently found in deep radio samples ${ }^{6}$. However it is still unclear whether they represent a substantial fraction of the entire FR I population that have been "hidden" by a selection bias, or they are only rare peculiar sources.

FR II: On average, FR II host galaxies are less luminous with respect to those of FR $\mathrm{Is}^{28}$ (but this is probably only because of selection effects ${ }^{40}$ ) and belong to lower density groups, at least at low redshifts ${ }^{45}$. One of the major unsolved issues for unification is the role played by a sub-class of lowionization FR II (LEG). Such objects have an FR II radio morphology, but their optical spectral properties are similar to those of FR I. Our analysis of HST images of the nuclei of $3 \mathrm{CR} \mathrm{FR} \mathrm{II}^{10}$ up to $z=0.3$ led us to intriguing conclusions. The first surprising result is that, as in the case of FR I, most of the galaxies show central unresolved components, regardless of their optical spectral classification. In the framework of the AGN unification scheme we would not expect to observe such optical nuclei in objects that do not show broad lines in their optical spectrum. In fact, their central regions should be hidden by the presence of thick obscuring tori. Broad-lined objects (quasars and broad line radio galaxies, BLO) have the brightest nuclei, which show a large optical excess with respect to the radio-optical correlation found for FR I (Fig. 2a). This is readily explained if the dominant component in the optical band is due to thermal emission from an accretion disk. Indeed, these nuclei appear to have flatter optical-to-UV spectral index $\left(\alpha_{o-U V} \lesssim 1\right)$ when compared to that of "synchrotron" FR I nuclei, and similar to that of other radio-loud $\mathrm{QSO}^{11}$. We also found that optical nuclei of BLO are present only for $L_{\mathrm{o}}>10^{28} \mathrm{erg} \mathrm{s}^{-1} \mathrm{~Hz}^{-1}$. This might be the manifestation of a threshold in the efficiency of the accretion process, from the standard optically thick, geometrically thin accretion disk to low radiative accretion flows.

The nature of the nuclei of the High Excitation Galaxies (HEG) is certainly more complex, since some of their nuclei may be compact scattering regions which fall on the FR I correlation "by chance". In order to discriminate between scattered nuclei and nuclei seen directly, we should refer to an isotropic parameter. The luminosity of the [OIII] emission line is believed to be a good indicator of the strength of the nuclear ionizing continuum. In Fig. 2b we show that sources separate in the plane formed by the "nuclear" EW of the [OIII] line vs the optical excess with respect to the non-thermal jet emission. BLO, LEG and FR I have low EW values $\left(\sim 10^{2.5} \AA\right)$ and 

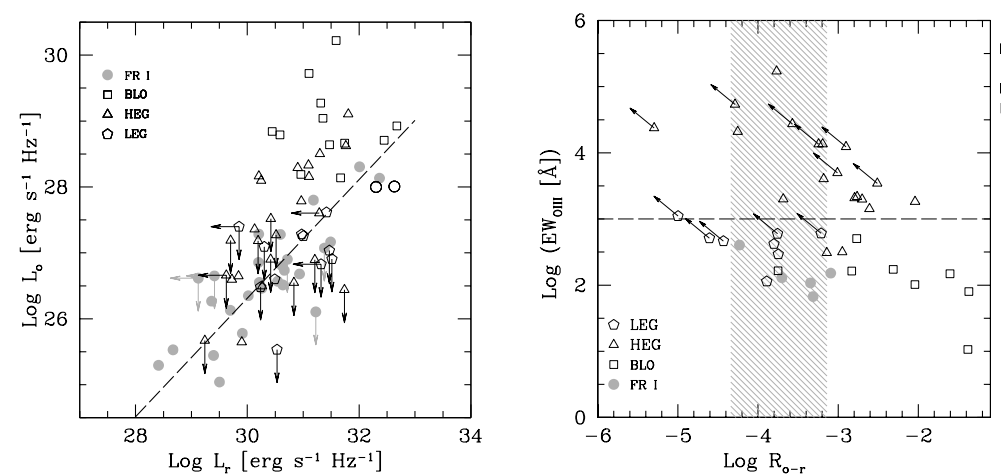

Figure 2. Left: (a) optical CCC vs. radio core luminosity for 3CR FR I and FR II with $z<0.3$. The dashed line is the FR I correlation Right: (b) Nuclear EW of the [OIII] emission line vs. the logarithm of the ratio between optical and radio core luminosity. The dashed lines separates scattered nuclei from nuclei seen directly.

they only differ by the amount of optical excess. On the other hand, all but two of the HEG have much larger equivalent widths ( $\gtrsim 10^{3.5} \AA$ ). This is indeed expected from the unified models, as obscuration reduces the observed nuclear continuum, while the line emission is less affected or unaffected. A strong ionization source, obscured to our line-of-sight, must be present in sources with a very high value of EW[OIII]. We argue that all sources with high EW are hidden quasars, and their nuclei are seen through scattered radiation, while the low EW region of the diagnostic plane includes the objects in which we directly see the source of ionization.

It is important to note that most of the LEG lie among the FR I in both diagnostic planes. Therefore, from the point of view of their radiooptical nuclear properties, these objects are indistinguishable from FR I. This picture leads to a new dichotomy for radio galaxies, which is not based on their radio morphology but on their nuclear properties. The LEGs should be considered as part of the same group as the FR I. A direct implication for the unifying scheme is that, when observed along the jet axis, LEGs should appear as BL Lac objects. Therefore, LEGs may be identified as the parent population of those BL Lacs with extended radio morphology ${ }^{26}$. Interestingly, both the evolution and unification of FR I and FR II with BL Lacs and flat-spectrum quasars (FSRQ) can be explained by a dual-population scheme ${ }^{24}$, which considers FR Is and LEGs as a single population, associated with BL Lacs, while all other FR II are unified to quasars. And our HST results basically confirm such a scenario. 


\section{Is there any radio-loud AGN sequence?}

The properties of the SED of blazars are well described by a single parameter, namely the bolometric luminosity ${ }^{18}$. As luminosity decreases, the frequency of the synchrotron peak increases as well as the ratio between the luminosity of the Synchrotron to inverse Compton peak. This characterizes the "blazars sequence", from FSRQ to low-energy-peaked BL Lacs (LBL) to high-energy-peaked BL Lacs (HBL), which have the synchrotron peak in the X-rays. Such a trend appears to be strictly connected with the intensity of the radiation field surrounding the relativistic jet ${ }^{22}$ : the position of the peaks of the SED is determined by the break of the emitting particles energy distribution, which is located at a lower energy for higher intensity radiation fields. This theoretical scenario implicitly predicts that high-energy-peaked blazars with strong emission lines (HFSRQ) should not exist. Recently, a population of HFSRQ might have been identified ${ }^{31}$. However, although their peak energy appears to be higher than that of "classic" FSRQ, $\nu_{\text {peak }}$ values are not as extreme as those of HBLs. This means that there may be a physical limit in $\nu_{\text {peak }}$ for sources with a strong radiation field surrounding the jet. Can we translate such a scenario into a physical sequence for radio galaxies? It is tempting to speculate on the existence of a similar sequence starting from low-power FR Is through HEGs (and BLRGs), with the LEGs acting as an intermediate class. But it is unclear if the differences between the various classes of RG are driven by e.g. a change in the physical conditions of the accretion process, which may also affect the properties of emission line field ${ }^{19}$. I believe this is one of the most interesting aspects of the unifying scheme for radio loud AGN to be investigated in years to come.

\section{Acknowledgments}

I apologize for the many omissions of my talk: the topic is immense, and it is impossible to be complete. Most of the work on radio galaxies' nuclei summarized here have been done with the fundamental contribution of other people. I wish to thank in particular Alessandro Capetti, Annalisa Celotti and Duccio Macchetto for productive collaboration and constant support. Finally, I wish to congratulate the LOC and the SOC, and in particular Raul and Roberto, for organizing a very successful meeting in such a beautiful place.

\section{References}

1. Antonucci, R. \& Barvainis, R. 1990, ApJ, 363, L17 
2. Antonucci, R. R. J. \& Miller, J. S. 1985, ApJ, 297, 621

3. Barth, A. J., Ho, L. C., \& Sargent, W. L. W. 2003, ApJ, 583, 134

4. Barthel, P. D. 1989, ApJ, 336, 606

5. Biretta, J. A., Sparks, W. B., \& Macchetto, F. 1999, ApJ, 520, 621

6. Blundell, K. M. \& Rawlings, S. 2001, ApJ, 562, L5

7. Capetti, A., Celotti, A., Chiaberge, M. et al. 2002, $A \& A$ A, 383, 104

8. Celotti, A., Ghisellini, G., \& Chiaberge, M. 2001, M.N.R.A.S., 321, L1

9. Chiaberge, M., Capetti, A., \& Celotti, A. 1999, A\&A, 349, 77

10. Chiaberge, M., Capetti, A., \& Celotti, A. 2002, A\&A, 394, 791

11. Chiaberge, M., Macchetto, F. D., Sparks, W. B., et al. 2002, ApJ, 571, 247

12. Chiaberge, M., Gilli, R., Capetti, A. et al. 2003, ApJ, 597, 166

13. Chiaberge, M., Capetti, A., \& Celotti, A. 2001, M.N.R.A.S., 324, L33

14. Cohen, M. H., Ogle, P. M., Tran, H. D. et al. 1999, AJ, 118, 1963

15. Corbett, E. A., Robinson, A., Axon, D. J., et al. 1996,M.N.R.A.S., 281, 737

16. Dunlop, J. S. et al. 2003, M.N.R.A.S., 340, 1095

17. Falomo, R., Kotilainen, J. K., \& Treves, A. 2002, ApJ, 569, L35

18. Fossati, G., Maraschi, L., Celotti, A. et al. 1998, M.N.R.A.S., 299, 433

19. Ghisellini, G. \& Celotti, A. 2001, $A \& A$, 379, L1

20. Giovannini, G., Cotton, W. D., Feretti et al. 2001, ApJ, 552, 508

21. Giovannini, G., Taylor, G. B., Arbizzani, E. et al. 1999, ApJ, 522, 101

22. Ghisellini, G., Celotti, A., Fossati, G. et al. 1998,M.N.R.A.S., 301, 451

23. Guainazzi, M., Grandi, P., Comastri, A. et al. 2003, $A \mathscr{E} A$, 410, 131

24. Jackson, C. A. \& Wall, J. V. 1999, M.N.R.A.S., 304, 160

25. Junor, W., Biretta, J. A., \& Livio, M. 1999, Nature, 401, 891

26. Kollgaard, R. I., Wardle, J. F. C., Roberts, D. H. et al. 1992, AJ, 104, 1687

27. Laing, R. A., Parma, P., de Ruiter, H. R. et al. 1999, M.N.R.A.S., 306, 513

28. Ledlow, M. J. \& Owen, F. N. 1996,AJ, 112, 9

29. McLure, R. J. \& Dunlop, J. S. 2002, M.N.R.A.S., 331, 795

30. Owen, F. N., Ledlow, M. J., \& Keel, W. C. 1996, AJ, 111, 53

31. Padovani, P., Perlman, E. S., Landt, H. et al. 2003, ApJ, 588, 128

32. Perlman, E. S., Sparks, W. B., Radomski, J. et al. 2001, ApJ, 561, L51

33. Scarpa, R., Urry, C. M., Falomo, R. et al. 2000, ApJ, 532, 740

34. Swain, M. R., Bridle, A. H., \& Baum, S. A. 1998, ApJ, 507, L29

35. Tadhunter, C. N. et al. 1993, M.N.R.A.S., 263, 999

36. Tavecchio, F., Maraschi, L., Sambruna, R. M. et al. 2000, ApJ, 544, L23

37. Tremaine, S., et al. 2002,ApJ, 574, 740

38. Urry, C. M. \& Padovani, P. 1995, PASP ,107, 803

39. Urry, C. M., Scarpa, R., O'Dowd, M. et al. 2000, ApJ, 532, 816

40. Scarpa, R. \& Urry, C. M. 2001, ApJ, 556, 749

41. Verdoes Kleijn, G. A. et al. 2002, AJ, 123, 1334

42. Whysong, D. \& Antonucci, R. 2004, ApJ, 602, 116

43. Willott, C. J. et al. 2000, M.N.R.A.S., 316, 449

44. Woo, J. \& Urry, C. M. 2002, ApJ, 581, L5

45. Zirbel, E. L. 1997, ApJ, 476, 489 